\title{
An Application-Layer Approach to Seamless Mobile Multimedia Communication
}

\author{
Johan Kristiansson and Peter Parnes
}

\begin{abstract}
Providing seamless IP mobility support is one of the most challenging problems towards a world of mobile and ubiquitous multimedia communication.

This paper proposes an application-layer framework based on the Session Initiation Protocol and the Resilient Mobile Socket to provide mobility support for distributed multimedia applications. As a part of the framework the paper describes a new handover strategy called Competition-based Soft-Handover Management that uses simulcast to improve quality of service and to seamlessly hand over multimedia traffic to the network interface that currently offers better network characteristics. In short, during a handover packets are simulcasted through all available network interfaces, and when the packets are received by the remote end-point, they are merged back into one stream. As each network connection competes with other connections in contributing to the merged stream at the remote end-point, the handover process can be viewed as a competition where the connection that contributes most is selected after the handover.

As a proof of concept, the framework has been integrated into Marratech, which is a commercially available audio/video group communication application. By using network emulators, the paper demonstrates how the framework can be used to improve QoS, compare end-to-end performance, and perform lossless handovers while reducing redundant packets.
\end{abstract}

Index Terms-Mobility management, Ubiquitous multimedia, Soft-handover

\section{INTRODUCTION}

The proliferation of mobile computing devices and deployment of wireless networks over the last decade have led to an increasingly nomadic computing lifestyle. Multimedia applications executing on portable devices allow users to be present and communicate with other users, anywhere, and anytime, through wide area cellular networks or inexpensive high performance wireless local area networks (WLAN) provided by a multitude of new wireless Internet service providers.

Allowing multimedia applications to take advantage of the wireless landscape requires that the system is able to detect the presence of wireless networks, configure the network interface (network card), and hand over the multimedia traffic to an appropriate access point. Similarly, the system must be able to hand over the traffic back to a wide-area carrier network before the connection is lost when the user is about to leave a network.

Research about mobility management has so far mainly focused on how to manage routing updates when users change point of attachment to the Internet. A general problem with today's mobility solutions is that they are hard to deploy and use in practice. Many existing solutions require dedicated

The authors are with the Department of Computer Science \& Electrical Engineering, Luleå University of Technology, 97187 Luleå, Sweden (e-mail: \{Johan.Kristiansson, Peter.Parnes\}@1tu.se). support from the network infrastructure; others require support from the operating system, thus limiting mobility support to only a few networks or a limited number of terminals. Another problem is that very few existing mobility protocols automatically handle handovers to other networks. For example, very few protocols can automatically switch from a WLAN to a UMTS network.

When developing an IP based handover algorithm, it is important to minimize packet loss and avoid introducing extra delay. A well designed handover algorithm should therefore be able to evaluate the performance of the available networks and select the best performing network as fast as possible in order to avoid interruptions in the communication. For example, even if a high performance WLAN is detected, the access point may not be usable, or be connected to low bandwidth backbone network providing worse performance than other available carriers. Hence, being able to compare end-to-end performance of available networks, and avoid accidentally switching to disconnected or misconfigured access points are both crucial.

Instability is another important problem that handover designers have to consider. If wireless performance fluctuates rapidly, for example due to radio interference, it is likely that handovers are triggered back and forth between two or more networks causing seriously degraded performance. This classical instability problem is known as the ping-pong problem [1], and it is particularly serious if it takes a long time to complete a handover as the total delay caused by handovers is the product of the time it takes to complete one handover and the handover frequency. These problems raise the question: Is it possible to design a mobility system that is easy to deploy and which automatically can evaluate end-to-end performance and perform handovers to the best network interface without the user perceiving interruptions in packet streams due to the handovers when using a real-time multimedia application?

To address the problem mentioned above, the paper proposes an application-layer framework based on the Resilient Mobile Socket (RMS) [2] and the Session Initiation Protocol (SIP) [3]. The paper also proposes a new handover decision algorithm called Competition-based Soft-Handover Management (CSHM) that automatically hands over the traffic to the most appropriate network interface that is available. While many factors can be considered when deciding the best network interface, this paper focus specifically on UDP based multimedia applications where delay, jitter, and packet loss are the most important criteria.

The rest of the paper is organized as follows. Section II 
gives a brief introduction to previous work related to mobility and handover management. In Section III, an overview of the framework is given followed by a more extensive description of the CSHM algorithm in Section IV. Section V describes a proof of concept prototype that has been built by integrating the framework into Marratech [4], which is a commercially available e-meeting application. Using this prototype several experiments are presented. In Section VI, the paper is finally concluded with a discussion and pointers to future work.

\section{BACKGROUND AND RELATED WORK}

There have been numerous proposals for adding mobility to IP based networks. This section summarizes some of these proposals and discusses the contribution of the proposed framework.

One of the most known mobility protocols for the Internet is Mobile IP [5]. By adding an indirection in the network layer, applications using Mobile IP can change point of attachment to the Internet while using a static home IP address that never changes. However, one of the main drawbacks of Mobile IP is that it requires dedicated support from the routers, or alternatively support from the users to setup and maintain tunnels, thus making it hard to deploy in practice.

To overcome some of the problems with Mobile IP, extensive work has been done to add mobility support in layers above the network layer [2], [6]-[8]. For example, the Stream Control Transmission Protocol (SCTP) [6] provides mobility support in the transport layer by using an add-on multi-homing management module [9].

In regards to multi-access handover control, Mark Stemm and Randy H. Katz [10] were among the first to consider simultaneous operation of multiple wireless network interfaces. They introduced the concept of wireless overlay networks, which is a hierarchy structure of network interfaces, where network interfaces that provide wider coverage is considered as higher level than network interfaces that only provide Internet connectivity in small areas. They also introduced the concept of vertical and horizontal handovers where vertical handovers occur when the user switches to another network interface, and horizontal handovers when the user switches between networks of the same type on the same sub network, for example changing default WLAN access point.

In most existing mobility architectures [10]-[13] handovers are triggered based on link layer information (e.g. signal strength). Although making handover decisions based on link layer information work well for making horizontal handovers, there are several problems when using them for making vertical handovers. First of all, because of the dynamic nature of wireless networks it can be difficult to compare link layer information obtained from various access technologies. Another problem is that there is no direct relationship between the signal strength and the actual performance of the network. In fact, even if the signal strength to an access point is good, the access point itself can be disconnected from the Internet, thus being useless except for communication between hosts located on the same sub network as the access point.

Wang et al. [1] describe a policy based handover control system that allows users to express a policy on what is the

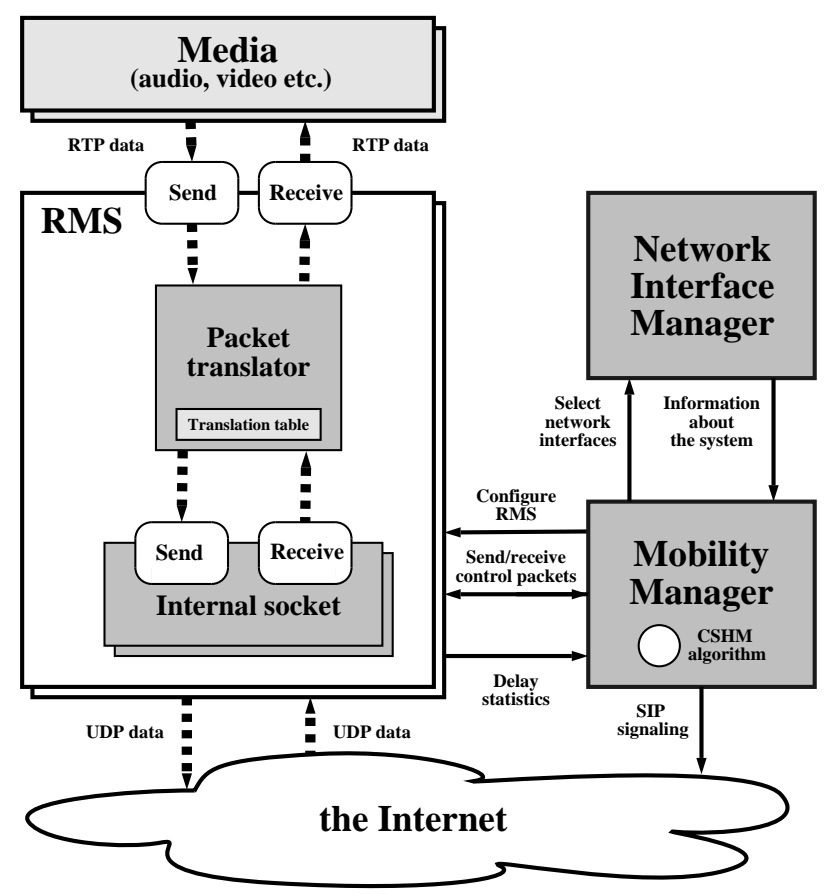

Fig. 1. Overview of the framework. All components shown in the figure reside in the application layer.

"best" network interface using more parameters than only signal strength. They also address the ping-pong problem by introducing a stability period, i.e. waiting a time before the handover is triggered and estimate if the new network is worthwhile to hand over to. A similar solution, which is used in many other handover control systems, is to use a combination of dwell-timers, delay or threshold based control algorithms [1], [11], [12] to make the handover control algorithm react more slowly to changes. However, for real-time multimedia communication it is better to perform a handover to a better network as fast as possible instead of waiting until the performance reaches a lower limit.

The major contribution of this paper is an applicationlayer framework that provides mobility support for UDP based multimedia applications. The Resilient Mobile Socket (RMS) has been introduced in a paper [2] previously published by the authors, but this paper contains a SIP extension to better handle mobility. The CSHM algorithm has also been introduced in a previously published paper [7]. This paper contains a more extensive description of the algorithm including an extension to better support vertical handovers. This paper also contains new unpublished experiments conducted using network emulators in addition to the real-life experiments presented in [7].

\section{APPLICATION-LAYER MOBILITY MANAGEMENT}

The Session Initiation Protocol (SIP) allows two or more participants to establish a session over the Internet. Schulzrinne et al. [8] describe how SIP can be used to provide mobility support at the application-layer by dynamically reestablishing sessions. They also introduce the concept of PreCall and Mid-Call mobility.

Pre-Call mobility occurs when a mobile client has moved to another network in prior to receiving or making a call. 
After the mobile client has obtained a new IP address, it reregisters with a SIP server allowing incoming invitations to be re-directed to the mobile client's current location.

Mid-Call mobility on the other hand occurs when the client moves to another network, or switches to another network interface during an already established session. After the mobile client has obtained a new IP address it re-invites the correspondent host in order to re-establish the communication. The framework presented in this paper can be viewed as an improved Mid-Call mobility scheme capable of utilizing multiple network interfaces simultaneously and automatically hand over to the one offering the least packet loss and delay. In general, Mid-Call mobility is more critical than Pre-Call mobility in e-meeting systems such as Marratech as users can participate in a session for a long period of time. As a reference, it can be mentioned that the authors are daily participating in a 24-hour connected e-corridor to share group location awareness.

Figure 1 shows an overview of the proposed framework. The framework consists of three main components, the mobility manager, the network interface manager, and the Resilient Mobile Socket (RMS). The RMS is an application layer mobility scheme used for Mid-Call mobility management. The mobility manager is responsible for deciding which network interface should be used, and monitors incoming packet streams to decide when to re-configure the remote RMS. It is also responsible for managing Pre-Call mobility and re-registering to a SIP server after a handover has been completed.

The third component, the network interface manager, deals primarily with configuration of network interfaces. It supplies the mobility manager with information about available network interfaces and is responsible for configuring the routing table and dealing with policy based routing in case the application wants to send and receive packets over multiple network interfaces at the same time. It is also responsible for notifying the mobility manager if it knows in advance that a network interface is going to be disconnected.

\section{A. Resilient Mobile Socket}

An application that sends and receives packets over the Internet normally uses a socket representing an end-point of a communication link to another application running on the Internet. By encapsulating multiple sockets into a new socket abstraction (i.e. RMS), any encapsulated or internal socket can fail without disturbing the applications. As each internal socket represents an entry point to each connected network, running applications will still be able to communicate if the currently active internal socket becomes disconnected and there is another internal socket available.

The advantage of using RMS versus a standard SIP solution is twofold. First of all, as mobility support is implemented in the socket-layer below the application, mobility support can simply be added by replacing the socket implementation. Secondly, adding an indirection in the socket layer allows duplication and filtering of both incoming and outgoing packets transparently to the application, which can be used to add simulcast and soft-handover support.
Packet translation is a key mechanism used by RMS to make a switch of an internal socket transparent and to ensure that packets are correctly routed. In short, the idea is to re-stamp all incoming packets according to a translation table so that it looks like they are coming from their original location and re-stamp all outgoing packets so that packets are redirected to hosts visiting foreign networks. From this point of view, the RMS almost works like a Network Address Translator (NAT), but on an application basis.

A fundamental mechanism to be able to keep the translation table updated is to be able to signal control information between RMS end-points. For example, in order to allow a remote end-point to re-route its outgoing traffic correctly to another internal socket, a handover request must be sent to the other end-point to let it update its translation table. RMS uses an in-band signaling protocol called Resilient Mobile Socket Control Protocol (RMSCP) to exchange control data between end-points. Control packets are injected in normal packet streams and separated from UDP data by examining the header of each packet. Packets that start with a unique 32 bits random number are treated as control packets and are forwarded to the mobility manager instead of the application.

The next section discusses how the mobility manager sends and receives control information in order to manage mobility.

\section{B. Mobility manager}

The main purpose of the mobility manager is to make sure that the application is using the best available network interface. Below is a short description of some strategies the mobility manager can use when selecting internal sockets.

Hard handovers: Handovers occur when it is better to switch to another internal socket than using the current one. If a mobile host has been previously disconnected and got back connectivity, its mobility manager sets up a new internal socket and sends an invite message to the mobility manager at the correspondent end-point to notify it about its new IP address. The correspondent end-point sends an OK message back, and updates its internal translation table to manage incoming packets correctly and ensure that outgoing packets are sent to the new internal socket at the sender. When the OK message is received by the mobile RMS end-point, it sends an acknowledgment message back and sets the new internal socket as default outgoing internal socket. Note that this scheme is very similar to the Mid-Call mobility procedure presented in [8], except that RMSCP is used to send control messages instead of SIP.

Soft-handovers: If the host has access to multiple network interfaces and the currently used network interface is likely going to be disconnected, it can make sense to make a preemptive handover to another network interface. This can be done by letting the mobility manager set up two internal sockets and send an invite message containing the address of each internal socket to the correspondent end-point. During the handover, packets are simulcasted through both the old and the new internal socket while switching to the new default internal socket specified in the invite message. Although softhandovers are particularly valuable as they allow a packet stream to be seamlessly migrated to another internal socket, 


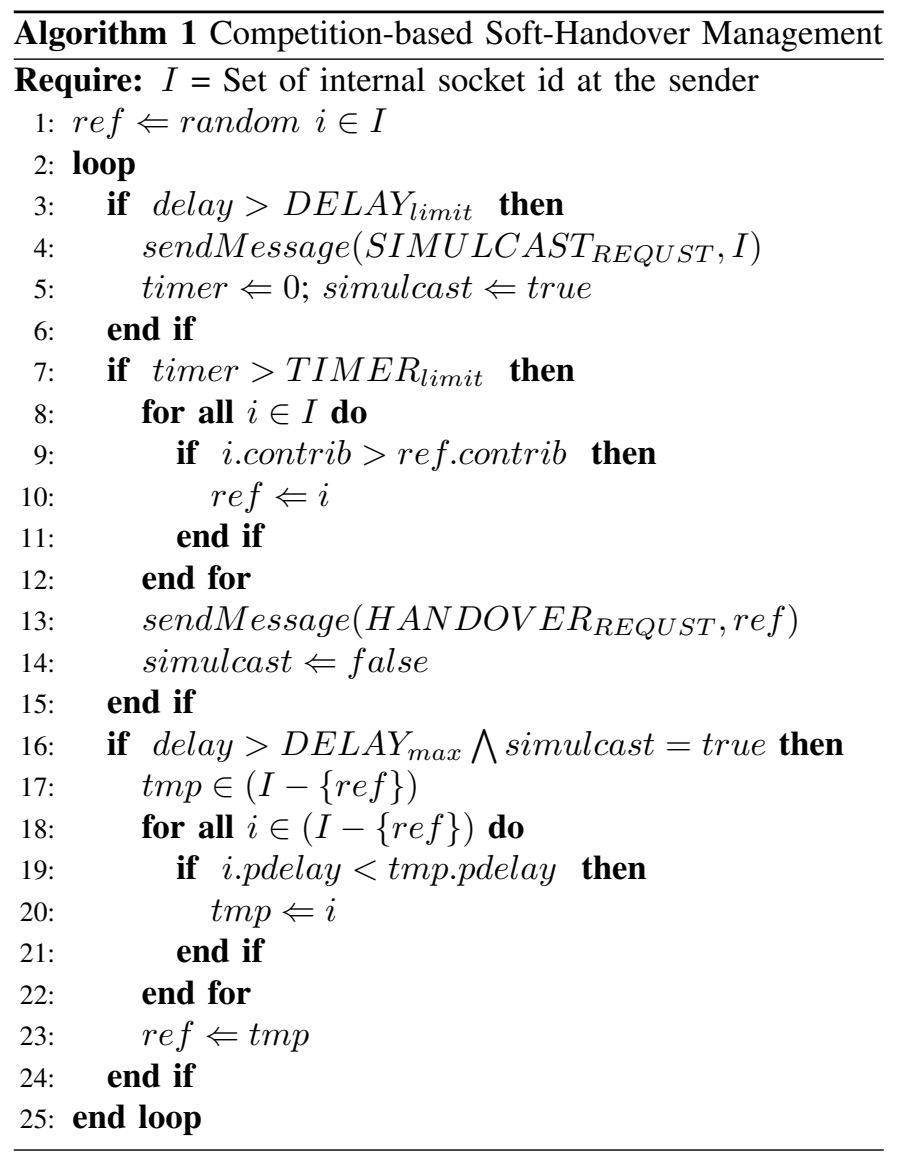

soft-handovers can be hard to trigger as they must be initialized proactively. Thus, some method is needed to predict when an internal socket is going to be disconnected in order to trigger a soft-handover. How the CSHM algorithm tackles this problem is discussed in Section IV.

Simulcast: If the host has access to multiple networks, it can also make sense to use simulcast to improve QoS. For example, sending important packets such as MPEG Iframes over redundant links in order to improve real-time video communication as suggested by [14], or duplicating all packets to improve audio quality as done in this paper. Moreover, to provide better bandwidth utilization and error resilience, another possibility can be to interleave outgoing data traffic. For example, sending every other packet on different internal sockets, or using different internal sockets for outgoing and incoming traffic. Yet another strategy to improve QoS can be to encode the traffic, for example XORing different combinations of packets. Nevertheless, one advantage of duplicating all packets is that it becomes possible to get separate network statistics for each duplicated packet stream, which can be used to compare end-to-end performance. The CSHM algorithm can be viewed as a combination of a softhandover and a simulcast algorithm as it uses simulcast to improve network performance but also to seamlessly hand over packet streams to another internal socket.

\section{COMPETITION-BASEd SOFT-HANDOVER MANAGEMENT}

As stated before, the purpose of the CSHM algorithm is to evaluate end-to-end performance and reduce packet loss and delay during handovers. It is primarily designed to support real-time multimedia communication, and is implemented as a plug-in to the mobility manager. The CSHM algorithm is summarized in Algorithm 1 and this section describes how it operates.

When radio conditions get worse, it is very common that packets get lost over the air interface. Link layer approaches such as automatic repeat request (ARQ) attempt to hide channel loss from the network layer by retransmitting lost packets. However, because it takes time to retransmit packets, ARQ tend to increase the packet delay, and as packets cannot be retransmitted forever some packets will still get lost. Consequently, when a user moves away from a wireless network physically, it is very likely that increased packet loss and packet delay occur just before a connection is completely lost. This information is used by the algorithm to enable simulcast.

The mobility manager at the receiving end-point is responsible for monitoring each connection and keeping statistics regarding which packet stream performs best. When a RMS end-point receives a packet stream from another RMS, it calculates a packet delay, delay, based on the arrival time of the current packet and the previously received packet. If the packet delay exceeds a threshold value, $D E L A Y_{\text {limit }}$, it sends a simulcast request message to the correspondent endpoint, asking it to start sending redundant packet streams using all available internal sockets simultaneously. This means that packet streams are simulcasted through all available network interfaces and when the packets are received they are merged back into one single stream. This process can be viewed as a competition as each internal socket competes with other internal sockets in contributing to the remote merged stream. The internal socket which delivers most packets to the merged stream wins the competition and is selected as the new default internal socket, $r e f$, after the performance has become satisfactory again.

One difference between traditional handover algorithms and the CSHM algorithm is that a new network is not decided before the handover is triggered, but is instead determined during the handover process. Another difference is that a handover can take place over a long period of time. In fact, if no individual connection can provide sufficient performance alone, the CSHM algorithm can also be used to improve QoS without actually hand over to another internal socket.

Several problems must be considered in order to use simulcast efficiently for handover management. First of all, some mechanism is needed to enable and disable simulcasting. Moreover, as simulcasting wastes resources both in terms of bandwidth and computer resources, an efficient simulcast algorithm must be able to minimize redundant packets while at the same time keep the QoS as good as possible. The rest of this section discusses how the CSHM algorithm addresses these problems and how handover decisions can be made by using a competition based comparison between internal sockets.

\section{A. Calculating the delay limit variable}

The delay limited, $D E L A Y_{\text {limit }}$, can be calculated in various ways. One way is to analyze packet delays from a previously received reference packet stream and study variations 
caused by the codec when minimal or no delay is introduced by the network. Normally, media encoders introduce some level of jitter as packets are not always generated periodically. For example, variable bit rate codecs such as H.261 generate packets depending on level of motion, bandwidth constraints, quality levels and so on. If the packet delay deviates significantly from an expected pattern, it can be assumed that the delay is caused by the network and a handover to another network can be initialized.

However, a problem with triggering handover based on interruptions in packet streams is that it can be hard to obtain a reference stream. One method is to train the system and calculate $D E L A Y_{\text {limit }}$ in advance for each configuration. The drawback of this method is that it requires user interaction. In addition, the system needs to be re-calibrated if a setting (e.g. video quality) is changed. Another method to calculate $D E L A Y_{\text {limit }}$ is to calculate it iteratively in real-time as the maximum value of all calculated packet delay values. This maximum value can be calculated by the sender RMS and sent over to the receiver end-point in order to avoid adding the delay introduced by the network.

Another problem with triggering handovers based on interruptions in packet streams is that many audio codecs apply silence suppression techniques to save bandwidth. Consequently, the CSHM algorithm will trigger a simulcast request even if the delay is caused by silence suppression and not the network. However, in this case, the sender end-point will not comply and reply with a decline message as it knows that the delay is not caused by the network. Silence suppression can be detected at the sender end-point simply by detecting that no packets have been sent to the RMS for some period of time, i.e. silence suppression is detected at the sender side and bad network performance is detected at the receiver side.

\section{B. Filtering out duplicate packets}

If packets are not lost over the network, the receiver will get duplicate copies from each simulcasted packet stream. To prevent this from happening, a mechanism is needed to filter out duplicate packets, and automatically disable simulcast when the performance becomes satisfactory again. By encapsulating all redundant packets into a new packet containing a sequence number, the first packet received for a given sequence number is forwarded to the application and all other copies are dropped. To prevent re-ordering of packets, old packets (i.e. the sequence number is less than the sequence number of the last received packet) are automatically dropped.

One advantage of using this first-come-first-serve scheme is that it can significantly improve the network performance during a handover. For example, if all available networks perform badly, it may still be possible to merge the networks into a better network.

\section{Deciding when to stop simulcasting}

To reduce redundant packets and to allow comparison between internal sockets, CSHM uses a dwell-timer that expires after a predefined amount of time, TIMER $R_{\text {limit }}$. Assuming that a new simulcast request has not been received (i.e. the dwell timer has not been reset), simulcasting will be disabled after the dwell-timer has expired.

During simulcasting, the mobility manager at the receiving end-point calculates in percentage how much each duplicated stream (internal socket) contributes to the merged stream. This new metric is called packet contribution (contrib), and can be viewed as a combination of packet loss and delay in respect to all other duplicated streams. The internal socket that got the highest packet contribution is selected as the new reference internal socket after the dwell-timer has expired. The mobility manager also sends a handover request message to the sending end-point asking it to stop simulcasting packets and switch to the internal socket specified in the message.

The whole handover process can be viewed as a competition where the threshold, DELAY limit, determines when the competition starts, the dwell-timer, TIMER $R_{\text {limit }}$, when the competition ends, and packet contribution who the winner is. As pointed out before, a competition may not necessarily result in a handover as it is possible that the currently selected internal socket wins. Consequently, the CSHM algorithm can also be used to improve network performance by decreasing network delay without actually switching to another network.

\section{Vertical handovers}

Different networks usually have different delay characteristics. For example, many of today's telecom networks usually have longer propagation delays than WLAN type of networks. This imposes a problem as different packet streams will arrive at different times, and can thus not always be used to compensate for packet loss. Two strategies can be used to tackle this problem. One strategy is to use the network with less propagation delay as the reference internal socket, and use the slower connection to repair packet loss. However, if the delay difference between the networks is too large and packets are dropped because they are too old, a more efficient strategy can be to use the slower network as reference internal socket and buffer packets from the faster network to compensate for packet loss. In general, the first strategy is preferable as the response time is reduced, but the second strategy is better if simulcast has no effect and the reference internal socket suffers from a severe loss rate.

In the CSHM algorithm, a new reference internal socket is selected if simulcast is enabled and if the packet delay exceeds a maximum delay limit, $D E L A Y_{\max }$. In this case, the algorithm selects the internal socket offering least propagation delay (pdelay). Note that no message is sent to the remote peer about the new reference internal socket as the remote peer should continue sending redundant packet streams.

\section{Evaluation}

To evaluate the framework and the CSHM algorithm, a proof of concept prototype was built by integrating the framework into Marratech [4], which is a commercially available emeeting software providing tools for synchronous interaction by combining audio, video, chat and a shared white-board. The following subsections explain how the prototype was implemented, describe the experiments, and present the results. 


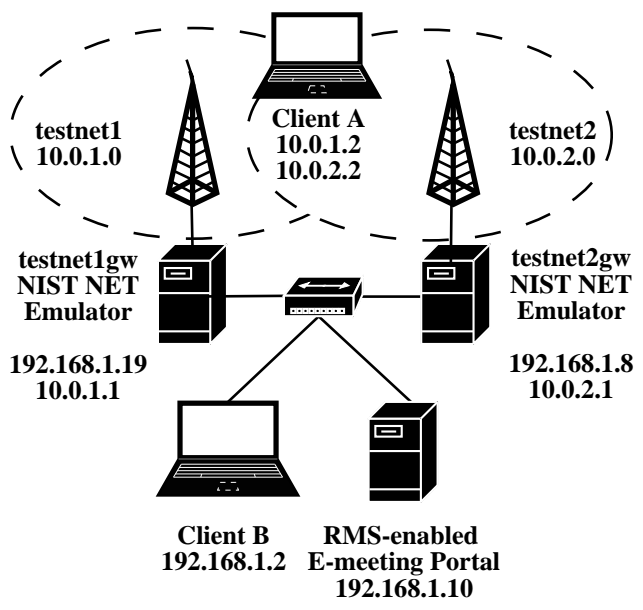

Fig. 2. The test-bed used in the experiments.

\section{A. Implementation}

The main part of the RMS and the mobility manager (including the CSHM algorithm) is implemented in Java JDK 1.5. Marratech was modified by replacing the standard Java DatagramSocket with the RMS. Since Marratech clients either use IP-multicast, or a media gateway called the E-meeting Manager to distribute packets to other participants, it was also necessary to replace the DatagramSocket implementation in the E-meeting Manager. The network interface manager is implemented in Java/C++ and uses a special NDIS driver available in the Java Wireless Research API [15] and the IP Helper API [16] to configure network interfaces. As the network interface manager is based on the NDIS framework, it can handle most network driver and card available in the Windows operating system. The NIST SIP implementation [17] was used to implement SIP support.

\section{B. Methodology}

One important objective with the experiments was to explore the CSHM parameter space in order to get a better understanding of how the DELAY limit, the DELAY $Y_{\max }$ and the $T I M E R_{\text {limit }}$ should be set. Other important objectives were to study bandwidth overheads, investigate how the CSHM algorithm performs using various media codecs, and investigate how network heterogeneity affected the performance.

Several experiments were conducted using real $802.11 \mathrm{~b}$ WLAN networks and a commercial GPRS network in order to test the prototype. However, it was difficult to repeat the same experiment and get the same result each test round as it was difficult to ensure the same mobility pattern and the same wireless conditions when re-running the same experiment. One solution to this problem would be to repeat the experiment until a statistical certainty is obtained, which unfortunately can make the experiments very time-expensive. Another problem using real networks is that it is difficult to study the effect of bandwidth and network latency variations as these parameters normally are fixed and can not be adjusted in a controlled way. One way to get around some of these problems is to use a network emulator to get more control over the networks. However, as very few network emulators can emulate wireless networks using configurable mobility patterns, trace files obtained from the real experiments were used to configure the network emulators.

Figure 2 illustrates the test-bed that was used throughout the experiments. The test-bed consists of two routers running the NIST Net network emulator [18]. Multimedia traffic was sent between two Marratech clients via the E-Meeting Manager. The first client (client A) used the framework whereas the second client (client B) did not use the framework and was only used to send and receive audio. Three computers were used during the experiments. The E-Meeting Manager was run on an AMD Athlon $1.2 \mathrm{GHz}$ machine running Windows XP. Client A and B were run on two Intel Pentium III $1.2 \mathrm{GHz}$ based machines running Windows XP. The network emulators were run on two Pentium-II $400 \mathrm{MHz}$ computers using the Linux 2.4.12 kernel. WLAN connectivity was provided by two Apple Airports with built-in NAT routing and two Lucent Orinoco 802.11b adapters attached to Client A. Each WLAN network interface was associated with different WLANs.

The algorithm was run every $10 \mathrm{~ms}$. To get better accuracy the Java Nano timer available in Java JDK 1.5 was used to generate time stamps in the collected log files, and each experiment (test-run) was run for 20 seconds and repeated 15 times. Error bars in figures show the standard error for each measurement.

The next subsection describes the real-word test and how the network emulator was configured. Subsection V-D investigates the CSHM parameter space, followed by an investigation in Subsection V-E on how network propagation delay affects packet aggregation. This experiment is followed up with an experiment about vertical handovers in Subsection V-F. In Subsection V-G it is investigated how the CSHM algorithm performs using various media codecs. Finally, in Subsection $\mathrm{V}-\mathrm{H}$ it is investigated how much bandwidth overhead is introduced by the control protocol.

\section{Experiment 1 - Real world test and emulator calibration}

This experiment was conducted by moving around physically in the test-bed with client A sending GSM audio between the two Marratech clients. This experiment was done earlier in a slightly different test-bed which is described in [7]. In this experiment, simulcasting was enabled during the whole experiment. Figure 3 shows the packet delay for each internal socket at Client A as well as the packet delay for the merged packet stream.

As can be seen in Figure 3(a) and 3(b), internal socket 1 lost connectivity three times while internal socket 2 lost connectivity only one time. Since all disconnections occurred at different times, it was possible to merge internal socket 1 and internal socket 2 to one packet stream (Figure 3(c)) without the user noticing any disconnections at all. Moreover, note that the packet delay for the merged stream is significantly reduced compared with internal socket 1 and internal socket 2. All copies of a specific packet were not always lost even if the packet loss rate was high for both internal sockets. However, the bandwidth overhead of using simulcast was $76 \%$ in comparison to only using internal socket 1 , or $89 \%$ in comparison to only using internal socket 2 . Hence, minimizing simulcasting to reduce bandwidth overhead is crucial. 


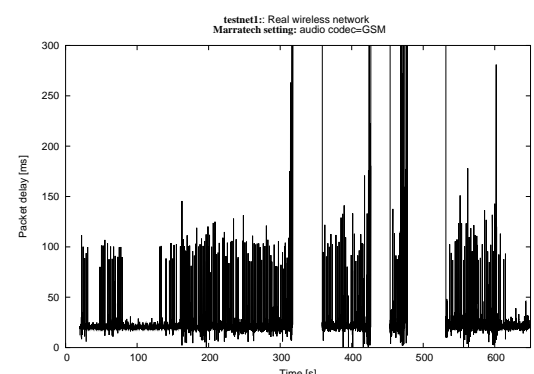

(a) Packet stream at ing internal socket 1 .

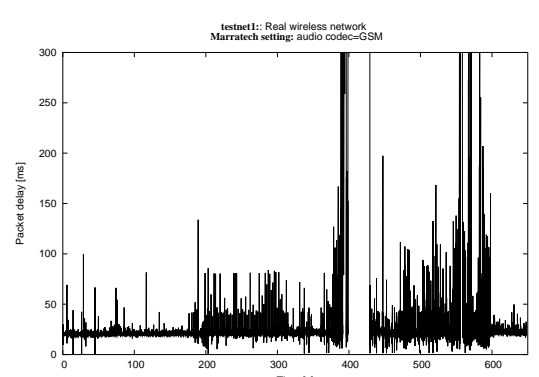

(b) Packet stream at inẹternal socket 2 .

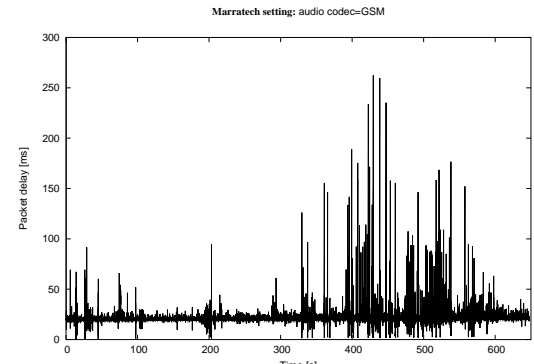

(c) Aggregated packet stream.

Fig. 3. Packet streams obtained from experiment 1.

Typically, when moving around client A in the test-bed, the current network performed optimally and then it was suddenly lost because the distance to the base station became too far. However, before the signal was completely lost, severe packet loss occurred, which was experienced as increased packet delay from an application point of view. By analyzing the recorded trace files from internal socket 1 it was found that $48 \%$ of all packets was lost in the time interval between $139 \mathrm{~s}$ and 294s. Additionally, an extra delay of $0.257 \mathrm{~s}$ was experienced during this time. Hence, by configuring the network emulator to randomly drop $48 \%$ of all packets and adding a small extra delay, it was possible to emulate a badly performing $802.11 \mathrm{~b}$ network. It is important to point out that this is a worst case scenario. In real life, successive packet loss and delay tend to be strongly correlated with each other whereas they are more evenly distributed when using the proposed network emulator settings. To compensate for this, the correlation factor for the delay and loss parameter was set to a maximum value of 0.8 . An alternative solution would have been to replay the trace files and re-configure the network emulator on the fly. However, as we were interested in studying how the algorithm performed when the networks performs badly, it was enough to only set the correlation factor for the delay and loss-rate parameters in the network emulator.

\section{Experiment 2 - Investigation into CSHM parameter space}

The second experiment was conducted to get a better understanding how the DELAY limit and the TIMER $R_{\text {limit }}$ parameter should be set. The CSHM parameter space was explored by locking one parameter, either $D E L A Y_{\text {limit }}$ or $T I M E R_{\text {limit }}$ and tuning the other parameter.

Both network simulators were configured as described in Section V-C, and client A and B send GSM traffic to each other. As can be seen in Figure 4, it was possible to reduce the packet loss by $25 \%$ by using a small $D E L A Y_{\text {limit }}$ value and setting the TIMER $R_{\text {limit }}$ to a value between 1000 and 2000 $\mathrm{ms}$. On the other hand, this resulted in more duplicate traffic as can be seen in Figure 5. Note that a packet loss reduction rate of $48 \%$ means that no packets at all were lost. From the diagram it can be seen that using a using a low TIMER $R_{\text {limit }}$ reduced the bandwidth overhead, but unfortunately it also reduced the packet loss reduction rate significantly. It can therefore be concluded that using a relative low DELAY limit value and a relative big TIMER $R_{\text {limit }}$ is optimal, which is

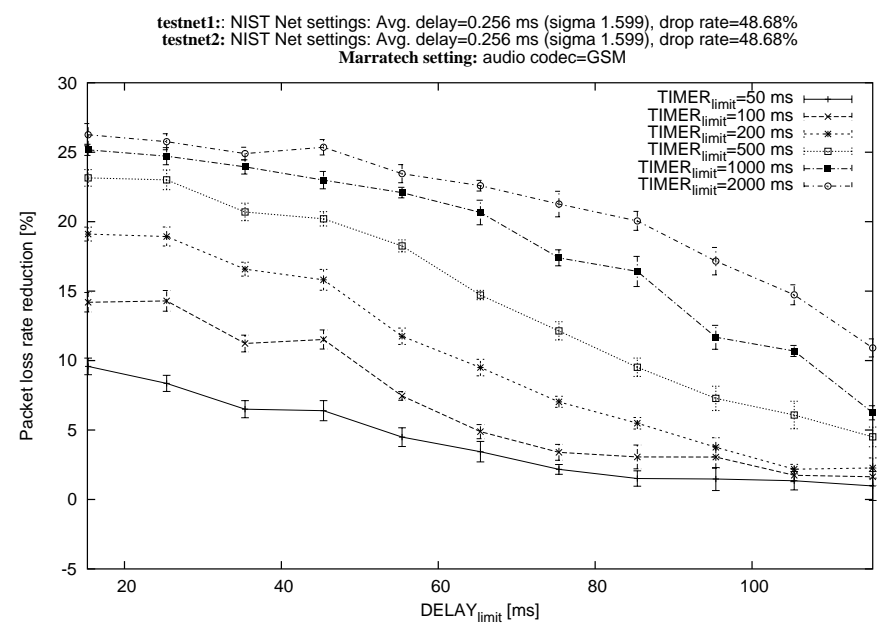

Fig. 4. Experiment 2: packet loss reduction rate.

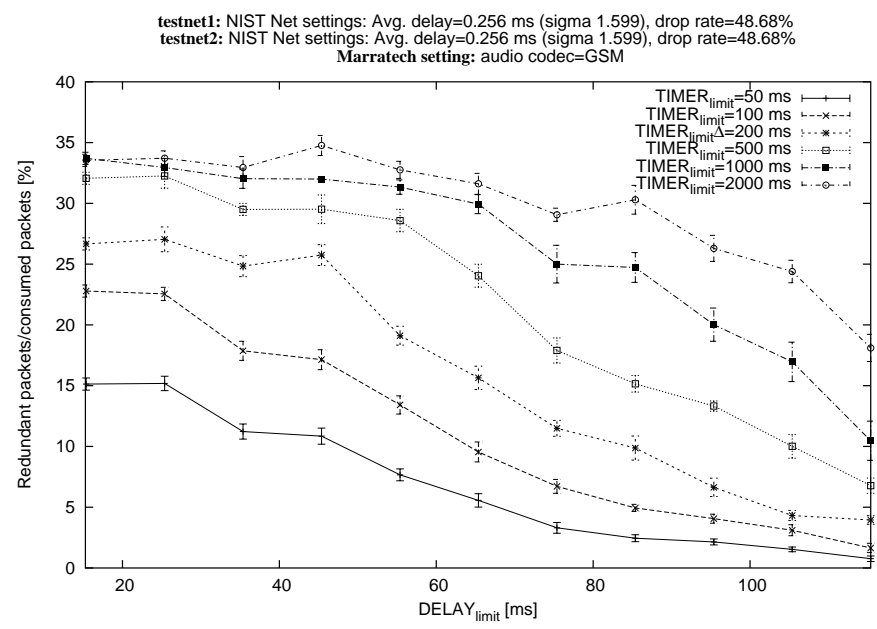

Fig. 5. Experiment 2: bandwidth overhead.

also consistent with results obtained from the previously done experiments [7]. However, even if these settings result in a significant amount of overhead, it should be pointed out that the experimental setup reflects a worst case scenario as both networks performed badly throughout the whole experiment. Bandwidth overhead caused by simulcasting will be much less if one of the networks performs well. 


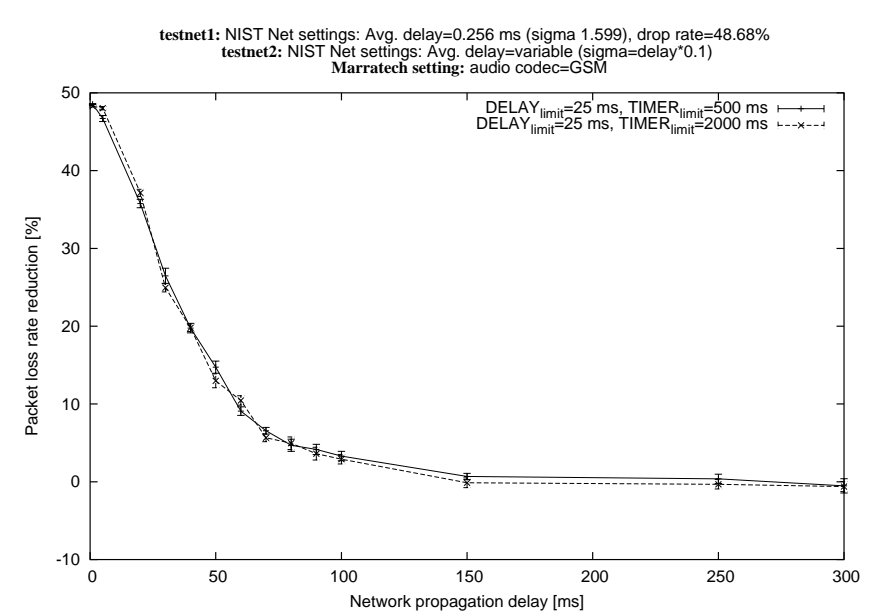

Fig. 6. Experiment 3: propagation delay implication.

\section{E. Experiment 3 - Propagation delay implication}

The third experiment was done to investigate how propagation delay variations affect the CSHM algorithm. A typical scenario would be using a low delay 802.11 network and a high-delay UMTS. The question is if it is possible to aggregate packet streams and reduce packet loss when there is a significant difference in packet propagation delay between the networks? To investigate this issue, one of the network emulator was configured according to the discussion in Subsection V-C. The other one was not configured to drop packets, but the propagation delay was varied from a small value of $0.1 \mathrm{~ms}$ to $300 \mathrm{~ms}$ during the experiment.

Figure 6 shows that the packet loss reduction rate is significantly reduced when the difference in propagation delay between the networks increases. This result suggests that it is not possible to aggregate packet streams from a commercial UMTS network (as it looks today) and a 802.11 type network. If the difference in delay is too large, it is better to use strategy 2 mentioned in Subsection IV-D, i.e. use the internal socket connected to the UMTS network as the reference internal socket and only use the 802.11 network as a backup network. This strategy is investigated further in the next experiment.

\section{F. Experiment 4 - Vertical handovers}

The fourth experiment was done to investigate if the performance can be improved by changing reference internal socket. The same experimental setup as experiment 3 was used, but the propagation was set to a static value of $300 \mathrm{~ms}$ during the whole experiment.

Figure 7 shows the packet delay variation during the experiment when using the internal socket connected to the lossy low delay network as reference. The network emulator causing the packet loss was enabled after 8 seconds. As packets from the high delay network were delivered too late, they could not be used to repair the stream. Figure 8 shows the same experiment but using the internal socket connected to the high delay network as reference. As can be seen, the performance was improved significantly except for the $300 \mathrm{~ms}$ peak which occur after 9 seconds. The peak is caused by the time it takes to catch up with the slower network.

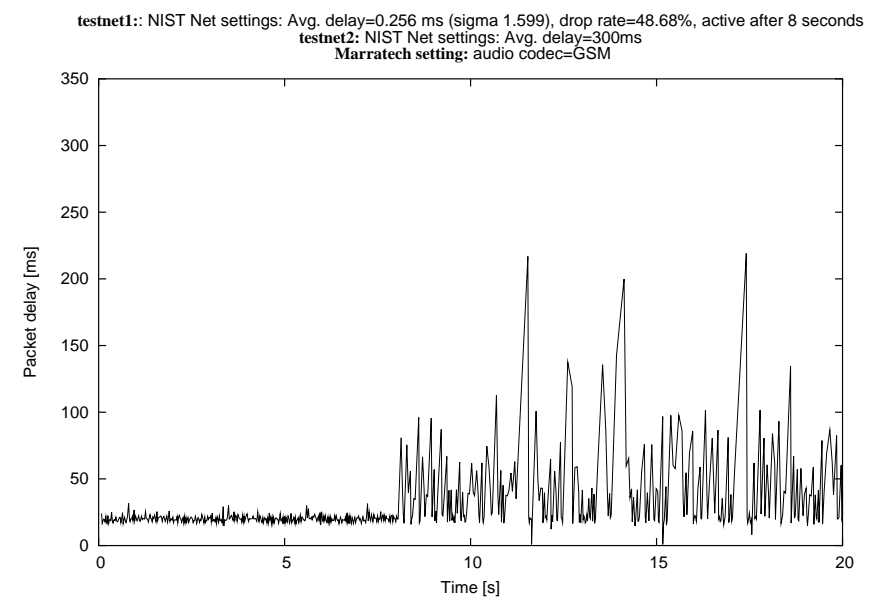

Fig. 7. Experiment 4: vertical handover using the lossy low delay network as reference. $D E L A Y_{\max }$ was set to $5 \mathrm{~s}$.

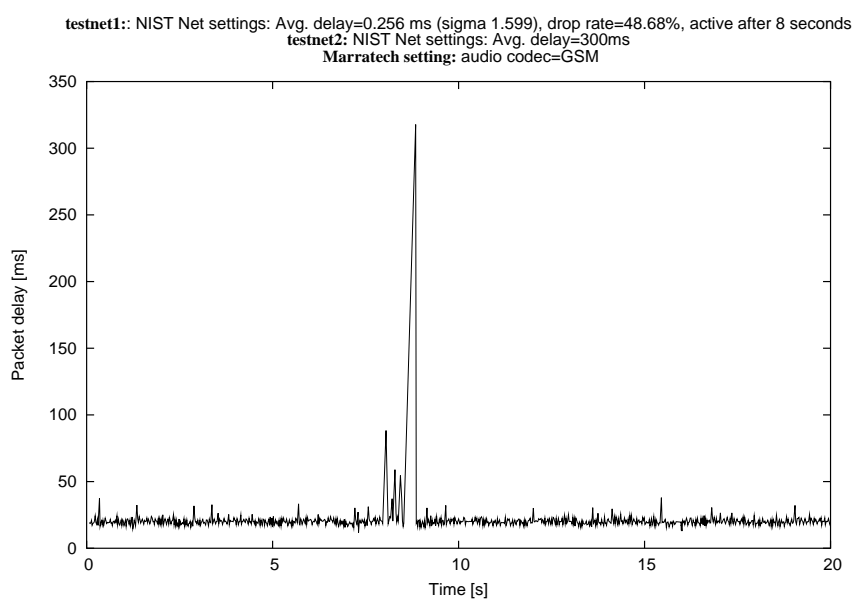

Fig. 8. Experiment 4: vertical handover using the high delay network as reference. $D E L A Y_{\max }$ was set to $90 \mathrm{~ms}$.

\section{G. Experiment 5 - Media encoding implication}

In all experiments so far a GSM codec has been used to generate data. This raises the question of how the CSHM algorithm performs using other media codecs, and also to what extent the results can be generalized? To answer this question, the same setup as in experiment 2 was used, but instead of varying the $D E L A Y_{\text {limit }}$ and the $T I M E R_{\text {limit }}$ parameter, each experiment was repeated using different media codecs.

Figure 9 shows the results from the experiment. As can be seen, there is no significant difference in packet loss reduction rate between the investigated codecs. However, as the H.261 codec uses variable bit rate encoding, it had a higher delay distribution during the experiment. Consequently, simulcasting was enabled all the time because of the high delay variation. This problem can be solved by setting a higher $D E L A Y_{\text {limit }}$ for the video traffic. Table I summarizes some statistics about the investigated codecs, such as the average packet delay and the delay variation interval. As can be seen in the table, for a $100 \mathrm{~kb} / \mathrm{s} \mathrm{H.261} \mathrm{codec,} \mathrm{the} \mathrm{DELAY} \mathrm{limit}$ should be set to at least $161 \mathrm{~ms}$.

Bandwidth overhead is another important difference that should be pointed out. From Table I it can be calculated that duplicating a GSM packet stream results in $3.5 \%$ bandwidth 


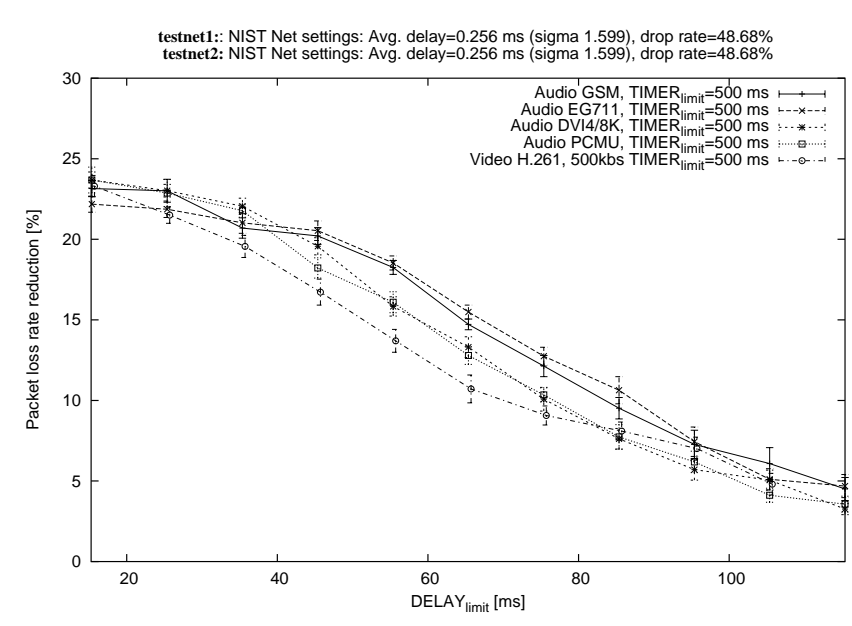

Fig. 9. Experiment 4: media encoding implication.

TABLE I

COMPARISON MEDIA CODECS.

\begin{tabular}{llll}
\hline Codec & $\overline{\text { delay }}$ & Delay interval & Bandwidth $^{a}$ \\
\hline H.261 $100 \mathrm{~kb} / \mathrm{s}$ & $61.47 \mathrm{~ms}$ & {$[16,161] \mathrm{ms}$} & $100 \mathrm{~kb} / \mathrm{s}$ \\
H.261 $500 \mathrm{~kb} / \mathrm{s}$ & $16.85 \mathrm{~ms}$ & {$[0.1,94] \mathrm{ms}$} & $500 \mathrm{~kb} / \mathrm{s}$ \\
H.261 $500 \mathrm{~kb} / \mathrm{s}$ (no motion) & $42.23 \mathrm{~ms}$ & {$[2,60] \mathrm{ms}$} & $500 \mathrm{~kb} / \mathrm{s}$ \\
GSM & $20.35 \mathrm{~ms}$ & {$[11,43] \mathrm{ms}$} & $13 \mathrm{~kb} / \mathrm{s}$ \\
DVI4/8K & $19.84 \mathrm{~ms}$ & {$[12,34] \mathrm{ms}$} & $33 \mathrm{~kb} / \mathrm{s}$ \\
PCMU & $19.96 \mathrm{~ms}$ & {$[11,43] \mathrm{ms}$} & $64 \mathrm{~kb} / \mathrm{s}$ \\
EG711 (GIPS) & $19.90 \mathrm{~ms}$ & {$[13,48] \mathrm{ms}$} & $79 \mathrm{~kb} / \mathrm{s}$ \\
\hline
\end{tabular}

${ }^{a}$ Bandwidth data in the table does not include IP/UDP/Ethernet headers

overhead when using a 384 kbps UMTS network. On the other hand, duplicating a packet stream generated by a EG711 codec [19] results in $20.6 \%$ overhead. Note that one advantage of the proposed framework is that different packet streams can be treated separately and only the most important streams (e.g. GSM traffic) need to be duplicated. It should also be pointed out that the main purpose of the CSHM algorithm is not to use simulcast for a longer period of time, but only for a short period of time during a handover to another network.

\section{H. Experiment 6 - Control protocol overhead}

The sixth experiment was conducted to study how much bandwidth overhead is introduced by the control protocol, and also if it is possible to use SIP instead of RMSCP to exchange control messages. The same settings as experiment 2 were used (20 seconds GSM data), but bandwidth overhead was studied instead of packet loss reduction rate. Attempts were also made to replace RMSCP with SIP. However, as the NIST SIP implementation sent SIP messages too slowly ${ }^{1}$, it was impossible to use it for exchanging control data. Nevertheless, it was possible to calculate how much bandwidth would have been consumed by SIP by analyzing the number of control packets sent.

Table II shows how the TIMER $R_{\text {limit }}$ variable affects the bandwidth overhead of the control protocol. As can be seen, $9.6 \%$ of the incoming traffic was control data when using RMSCP, and $77 \%$ when using SIP. Table III shows how

\footnotetext{
${ }^{1}$ It took in average 1.5 seconds to send a message. This delay is most likely caused by the NIST SIP stack and not by the protocol standard.
}

TABLE II

BANDWIDTH OVERHEAD COMPARISON. DELAY limit WAS SET TO 25 MS.

\begin{tabular}{lll}
\hline TIMER $R_{\text {limit }}$ & Overhead SIP & Overhead RMSCP \\
\hline 50 & $77 \%$ & $9.6 \%$ \\
100 & $72 \%$ & $7.7 \%$ \\
200 & $67 \%$ & $6.3 \%$ \\
500 & $62 \%$ & $5.0 \%$ \\
1000 & $61 \%$ & $4.7 \%$ \\
2000 & $60 \%$ & $4.6 \%$ \\
\hline
\end{tabular}

TABLE III

BANDWIDTH OVERHEAD COMPARISON. TIME $R_{\text {limit }}$ WAS SET TO 500 MS.

\begin{tabular}{lll}
\hline$D E L A Y_{\text {limit }}$ & Overhead SIP & Overhead RMSCP \\
\hline 0 & $61.3 \%$ & $4.8 \%$ \\
10 & $49.6 \%$ & $3.0 \%$ \\
20 & $42.5 \%$ & $2.3 \%$ \\
30 & $34.2 \%$ & $1.6 \%$ \\
40 & $31.1 \%$ & $1.4 \%$ \\
50 & $23.8 \%$ & $0.1 \%$ \\
60 & $18.2 \%$ & $0.07 \%$ \\
\hline
\end{tabular}

bandwidth consumed by control data can be decreased by using a larger $D E L A Y_{\text {limit }}$. These results can be explained by the fact that a minimal SIP packet is 342 bytes large whereas a RMSCP packet is only 11 bytes large. Considering that SIP introduces a significant amount of bandwidth overhead, the paper recommends RMSCP for exchanging control data between RMS end-points and SIP for managing Pre-Call mobility.

\section{DISCUSSION}

In the introduction the following question was asked:

Is it possible to design a mobility system that is easy to deploy and which automatically can evaluate end-to-end performance and perform handovers to the best network interface without the user perceiving interruptions in packet streams due to the handovers when using a real-time multimedia application?

To solve this problem, the paper has presented an application-layer mobility framework based on SIP and RMS to allow applications to efficiently switch between network connections. The paper has also presented a handover strategy called CSHM that uses simulcast to seamlessly handover a packet stream to another network interface. The paper has shown how simulcast can be used to compare end-to-end performance of available connections in order to figure out which network interface is best suitable for real-time communication. Moreover, the paper has shown how simulcast can efficiently be used to improve QoS by reducing packet loss and endto-end delay over wireless links. In some situations, when the difference in propagation delay is small, it may even be possible to combine two badly performing networks into one good performing network as confirmed by the experiments. However, if the delay difference is too large, it is better to hand over to the slower network.

In regards to the ping-pong problem, the CSHM algorithm does not directly eliminate oscillations as it is still possible that 
handovers (simulcast requests) are triggered back and forth between several networks. However, the user will not perceive degraded performance due to the handovers as the application receives packets from both the old and the new network during the handover. As a result, it is no longer important to reduce the handover frequency, or avoid handovers to WLANs with uncertain performance. This property is in particular useful when connecting to a new WLAN where the performance or QoS is not known in advance. However, it should be pointed out that sending a large amount of simulcast requests will result in increased bandwidth utilization (especially when using SIP). Thus, the paper recommends RMS and RMSCP for Mid-Call mobility and SIP for Pre-Call mobility when using the CSHM algorithm.

To trigger handovers, the paper has proposed a method based on detecting disruptions in packet streams. This allows handovers to be triggered promptly, but in the current version of the framework, the delay limit must be estimated by the user. Ultimately, the delay limit should be calculated adaptively based on the current bit-rate, the level of quality and the type of codec being used. The CSHM algorithm can also be further improved by better supporting vertical handovers. In the current version, the connection with least propagation delay is selected, but there is no way to change back to the previously reference internal socket except if the new connection starts to perform badly. The main reason for this limitation is to minimize the delay introduced when changing reference internal socket. However, an interesting method might be to trigger handovers during silence periods, for example when the user is not speaking.

To summarize, the paper has presented an applicationlayer mobility framework. By updating the socket it becomes possible to preserve connectivity, triggering handovers by using information obtained from packet streams, and reduce packet loss during handovers by simulcasting important packet streams while at the same time requiring minimal modification to the application, thus making it easy to deploy.

\section{ACKNOWLEDGMENT}

This work was done within the VITAL and the $\mathrm{C} 4$ project, which are supported by the Objective 1 Norra Norrland - EU structural fund programme. Support was also provided by the Centre for Distance-spanning Technology (CDT). Finally, the authors would like to thank the anonymous reviewers for their valuable comments.

\section{REFERENCES}

[1] H. J. Wang, R. H. Katz, and J. Giese, "Policy-Enabled Handoffs Across Heterogeneous Wireless Networks," in IEEE Workshop on Mobile Computer Systems and Applications, 1999, pp. 51-60.

[2] J. Kristiansson and P. Parnes, "Application-layer Mobility support for Streaming Real-time Media," in IEEE Wireless Communications and Networking Conference (WCNC'04), 2004.

[3] J. Rosenberg, H. Schulzrinne, G. Camarillo, A. Johnston, J. Peterson, R. Sparks, M. Handley, and E. Schooler, "SIP: Session Initiation Protocol," June 2002, IETF RFC3261.

[4] Marratech AB, 2005, <http://www.marratech.com>.
[5] C. Perkins, "IP Mobility Support," 1996, IETF RFC2002.

[6] R. S. et al., "Stream Control Transport Protocol," 2001, IETF RFC2960.

[7] J. Kristiansson and P. Parnes, "Providing Seamless Mobility with Competition based Soft Handover Management," in LNCS 3271, 7th IFIP/IEEE International Conference on Management of Multimedia Networks and Services, MMNS, 2004, pp. 295-307.

[8] H. Schulzrinne and E. Wedlund, "Application-Layer Mobility Using SIP," ACM SIGMOBILE Mobile Computing and Communications Review, vol. 4, no. 3, pp. 47-57, 2000.

[9] R. Steward and et al., "Stream Control Transmission Protocol (SCTP) Dynamic Address Reconfiguration,” 2003, Internet Draft, IETF. Work in progress.

[10] M. Stemm and R. H. Katz, "Vertical Handoffs in Wireless Overlay Networks," Mobile Networks and Applications, vol. 3, no. 4, pp. 335350, 1998.

[11] S. Aust, D. Proetel, N. A. Fikouras, and C. Görg, "Policy based Mobile IP Handoff Decision (POLIMAND) using Generic link Layer Information," in IEEE 5th International Conference on Mobile and Wireless Communication Networks (MWCN'02), 2003.

[12] Y. Min-hua, L. Yu, and Z. Hui-min, "The Mobile IP Handoff Between Hybrid Networks," in IEEE 13th International Symposium on Personal, Indoor and Mobile Radio Communication (PIMRC'02), 2002, pp. 256269.

[13] S. Sharmaa, I. Baek, Y. Dodia, and T. Chiueh, "OmniCon: A Mobile IP-Based Vertical Handoff System for Wireless LAN and GPRS Links," in International Conference on Parallel Processing Workshops (ICPPW'04), 2004, pp. 330-337.

[14] A. A. E. Al, T. Saadawi, and M. Le, "Improving Interactive Video in Wireless Networks Using Path Diversity," in LNCS 3271, 7th IFIP/IEEE International Conference on Management of Multimedia Networks and Services, MMNS, 2004, pp. 1-12.

[15] J. Kristiansson, "Java Wireless Research API," 2005, $<$ http://www.csee.ltu.se/ johank/javawrapi/>.

[16] Microsoft IP Helper API, 2005, <http://msdn.microsoft.com $>$.

[17] National Institute of Standards and Technology., "NIST SIP," 2005, $<$ http://http://snad.ncsl.nist.gov/proj/iptel $>$.

[18] M. Carson and D. Santay, "NIST Net: a Linux-based network emulation tool," ACM SIGCOMM Computer Communication Review, vol. 33, no. 3, pp. 111-126, 2003.

[19] Global IP Sound AB, 2005, <http://www.globalipsound.com>.

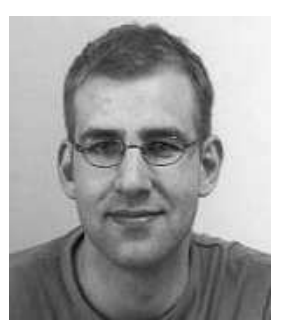

Johan Kristiansson received the MSc degree in Computing Science and Engineering from Umeå University, Umeå, Sweden, in 2000. From 2000 to 2002, he worked as a software engineer at Marratech $\mathrm{AB}$ where he developed video conferencing and emeeting softwares for the Internet. From 2002 he has been working toward the $\mathrm{PhD}$ degree in $\mathrm{Me}$ dia technology at Luleå University of Technology, Luleå, Sweden. In 2004 he got the Licentiate in Technology degree. His research interests are in mobility management, always-best-connected services, context-awareness, and bandwidth management in multimedia systems.

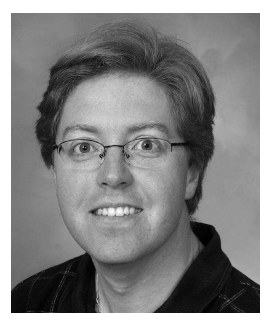

Peter Parnes is a researcher, who enjoys applied research which is attractive to the industry outside the research community. Research interests include distributed applications and real-time human communication over the Internet. Peter Parnes has a $\mathrm{PhD}$ in Computer Science received from the Luleå University of Technology in 1999 and he became Associate Professor at the same university in 2004. He has a strong record of financing research and he has insight into how to create spin-off companies as he is the founder of the Swedish company Marratech AB. Peter holds five patents in the area of distributed applications and he has published more than 40 academic publications in international journals and conferences. Peter Parnes is currently employed at Luleå University of Technology, where he leads research in Media Technology, as well as at Marratech $\mathrm{AB}$ as Chief Scientist. Peter Parnes has a long experience in creating and leading research projects and he strongly believes that applied research leads to both stronger academic results as well as results that are attractive to the industry, which in turn contributes to business development. 